Stoler, Ann Laura, Carnal Knowledge and Imperial Power. Race and the Intimate in Colonial Rule, Berkeley 2002.

Terkessidis, Mark, Interkultur, Berlin 2010.

Wollrad, Eske, »Kolonialrassistische Stereotype und Weiße Dominanz in der PippiLangstrumpf-Trilogie«, in: Benz, Wolfgang (Hg.), Vorurteile in der Kinder-und Jugendliteratur, Berlin 2010, S. 63-77.

Winkler, Peter, »Beim kirgisischen Bruder des Petit Suisse. >Schwejzartschik< aus der Milchquelle am Issyk-Kul«, in: Neue Zürcher Zeitung vom 03.10.2005, http://www.nzz.ch/2005/10/03/al/articleD6VSY.html, 24.01.2012.

\title{
ABBILDUNGSNACHWEISE
}

Abb. $1 \quad G l o b i$ Verlag, Globis Weltreise, Zürich 1935, o.S.

Abb. 2 Globi Verlag, Globis Weltreise, Zürich 1935, o.S.

Abb. 3 Plattencover von Kasperli Nr. 7, De Schorsch Gaggo reist uf Afrika, Zürich Ex Libris [1970]

Abb. 4 Globi Verlag, Globis Weltreise, Zürich 1935, o.S.

Abb. 5 Globi Verlag, Globi bei den Nashörnern, Zürich 2007, S. 29

Abb. 6 Globi Verlag, Globi bei den Nashörnern, Zürich 2007, S. 31

Abb. 7 Globi Verlag, Globi bei den Nashörnern, Zürich 2007, S. 31

\section{Geschlecht im Schweizer Migrationsdiskurs}

Die postkoloniale Konstruktion der "unterdrückten Muslimin" und die rassistische Verwendung des Schleiers

\section{Meral Kaya}

Die Parlamente Belgiens und Frankreichs haben sich im Jahr 2010 für ein Gesetz ausgesprochen, welches das Tragen eines Ganzkörperschleiers im öffentlichen Raum verbieten soll. Auch in der Schweiz wurde seit dem Erfolg der Minarettinitiative heftig über das Phänomen des Ganzkörperschleiers diskutiert. ${ }^{1}$ So forderte der Kanton Aargau mit einer Standesinitiative ein nationales Burkaverbot, und im Tessin wird ein Verhüllungsverbot im Rahmen einer kantonalen Volksinitiative zur Abstimmung kommen. ${ }^{2}$ In der Schweiz aber gibt es kaum Frauen, welche einen Ganzkörperschleier tragen ${ }^{3}$, genauso wenig in Belgien, wo die Zahl der sogenannten Ganzkörperschleierträgerinnen auf einige Dutzende geschätzt wird. In Frankreich kommen diese vor kurzem in die Öffentlichkeit katapultierten Frauen auf die Zahl von 2000.4 Gemeinsam ist den Verbotsbefürworterinnen und -befürwortern in diesen drei Ländern das vermeintliche Hauptmotiv in der Debatte,

1 | Aus medienwirksamen Gründen wird dieser Schleier auch fälschlicherweise Burka genannt, obwohi das Tragen der Burka in Europa kaum verbreitet ist. Die Burka ist ein Schleier, welcher den ganzen Körper und das Gesicht verhüllt. Die Augenpartie wird dabei zusätzlich durch ein Stoffgitter bedeckt. In Europa häufiger anzutreffen sind der Tschador oder das Niqab, oft in Kombination miteinander getragen. Der Tschador ist ein schwarzes Tuch, welches als Umhang um Körper und Kopf getragen wird. Das Gesicht bleibt dabei frei. Das Niqab ist ein Gesichtsschleier, weicher bloß die Augenpartie freilässt. Die falsche Begrifflichkeit zeigt den symbolischen Gehalt der Debatte, welche nicht den Anspruch hat, auf wahrheitsgetreuen Fakten zu basieren. Ich vermeide im vorliegenden Artikel den Begriff der Burka bewusst.

2 | N. N., "Burkaverbot wird Abstimmungsthema", in: Neue Zürcher Zeitung Online vom 19.05.2011

3 | Hollenstein, "Widmer-Schlumpf erwägt Burka-Verbot», in: Neue Zürcher Zeitung Online vom 08.11.2009.

4 | Baspinar, Deniz, "Burkaverbot, einig Vaterland «, in: Die Zeit Online vom 01.05.2010. 
nämlich die Gleichstellung der Geschlechter. Im Folgenden soll gezeigt werden, dass sich diese Diskussion bei näherer Betrachtung als eine Stellvertreterdebatte erweist. Unter dem Vorwand der Geschlechtergleichheit wird in diesen Debatten vor allem eine harte Linie in der Migrations- und Integrationspolitik legitimiert.

In diesem Artikel wird aufgezeigt, dass die Art und Weise, wie die Debatten zu Kopftuch und Ganzkörperschleier geführt werden, kolonialer Natur sind. Dabei zeigen sich nochmals viele Aspekte, die Edward Said bereits 1978 in seinem Werk Orientalismus aufgezeigt hat. Der Orient, laut Said eines der ältesten Kolonialgebiete Europas, ist demnach eine Erfindung der europäischen Kultur. ${ }^{5}$ Bei dem Diskurs über den Orient handelt es sich um einen spezifisch westlichen Stil der Herrschaft, eine Autoritätsausübung gegenüber dem kolonialen Anderen, die sich unter anderem wissenschaftlicher Mittel bedient. Dadurch, dass das vermeintlich Fremde der eigenen Kultur, der Orient dem Okzident, gegenübergestellt und als unterlegen dargestellt wird, gewinnt die europäische Kultur an Stärke und Identität. ${ }^{6}$ Die Überlegenheit der eigenen Kultur wird insbesondere durch Bevormundung durchgesetzt. So werden Verbote, Gesetze und Zurechtweisungen zur Voraussetzung der Anpassung an die westliche Kultur. Dieser Prozess kann auch in gegenwärtigen Gesellschaften beobachtet werden und kommt insbesondere in den sogenannten Integrationsvorstellungen zum Ausdruck. Dieser koloniale Hintergrund macht erst deutlich, warum ein Stück Stoff, das zum Symbol der Unterdrückung der muslimischen Frau geworden ist, so viel Aufruhr verursacht und verboten werden soll. Der gegenwärtige Diskurs zur »Befreiung« muslimischer Frauen wird jedoch nicht - wie man annehmen könnte - bloß von altbekannten rechtspopulistischen Akteuren und Parteien geführt, er wird auch von linken und feministischen Gruppen sowie der Gesellschaft im Allgemeinen getragen. Diesen unterschiedlichen Akteuren und Akteurinnen entsprechend liegt der Fokus dieses Artikels zum einen auf den Debatten in einer breiten Öffentlichkeit, dem Mainstream, weil diese am meisten gehört und durch die Medien verbreitet werden. Zum anderen wird sich der Blick auf die Rolle von gewissen westeuropäischen Feministinnen richten und veranschaulichen, inwiefern ein islamophober Diskurs diesen zugutekommt. Schließlich soll auch auf die wenig gehörten kolonialismuskritischen feministischen Gegenstimmen eingegangen werden.

\section{Dominanz der Mehrheitsgesellschaft}

Um diese Debatten über die muslimische Kultur und die von ihr ausgehende Unterdrückung der muslimischen Frau verstehen zu können, müssen in einem ersten Schritt die hierarchisch aufgebaute Schweizer Gesellschaft und deren Machtverhältnisse betrachtet werden. Der Diskurs in Politik, Wissenschaft und in

5 | Said, Orientalismus, S. 8.

6 | Ebd., S. 10 den Medien vermittelt eine spezifische Vorstellung von Kultur, die als eine homogene, statische und nationale Einheit betrachtet wird. Durch Migration und Differenz, das heißt durch die vermeintlich pathologische und fremde Kultur, die »eindringt«, wird nach dieser Logik die Ordnung und Stabilität der Gesellschaft gefährdet. $^{7}$ Die Reduktion von bestimmten Migrantinnen und Migranten auf eine spezifisch andere Kultur setzt einen sozialen Exklusionsmechanismus in Gang, welcher Minderheiten schafft, diese (fast immer negativ) etikettiert und gleichzeitig die Privilegien einer dominanten Mehrheit zementiert. ${ }^{8}$ Ausgrenzung und Diskriminierung sind die Folgen dieser Zuschreibungsprozesse, die mit Bezug auf Ethnizität und Geschlecht đurch die Dominanzgesellschaft ausgeführt werden. Im Verlauf dieses Prozesses wird die eigene Gruppe kontinuierlich aufgewertet, während die Gruppe der Migrierten abgewertet wird. ${ }^{9}$ Zudem werden Eigen- und Fremdgruppen nach Kriterien definiert, die als »natürlich« und dadurch nicht veränderbar angesehen werden. ${ }^{10} \mathrm{Im}$ Zuge dieser Aufwertung wird die Machtposition derjenigen dominanten Gruppe nochmals unterstrichen, welche die territorialen und nationalstaatlichen Rechte beansprucht." Ein zentraler Effekt dieser Logik ist, dass die sogenannten Integrationsprobleme von Migrantinnen und Migranten diesen selbst zugeschrieben werden können. Die Migrationspolitiken des eigenen Staates hingegen geraten fast nie in Kritik. Anstatt die strukturellen Dimensionen der sogenannten Integrationsproblematik zu thematisieren, welche die ganze Gesellschaft betreffen, werden Sündenböcke kreiert, die von der eigenen Mitschuld und Verantwortung ablenken. Eine solche Sichtweise fordert von einer zugewanderten Minderheit, sich dem Lebensstil der Dominanzgesellschaft anzupassen. In dieser einseitigen Angleichung zeigt sich der Universalitätsanspruch Letzterer, sprich der Eurozentrismus des Westens. ${ }^{12}$

In dieser eurozentrischen Tradition kann auch der aktuelle Diskurs über den Islam verortet werden. Tendenziell wird alles, was nicht christlich-abendländisch ist, als eine der eigenen Kultur unterlegene Lebensweise betrachtet. Diese Einschätzung ist ein Produkt der langen und vom Kolonialismus geprägten Beziehung von Okzident und Orient, einer Beziehung, die von Macht, Herrschaft und Hegemonie gezeichnet ist. ${ }^{13}$ Dazu gehört auch die Produktion einer Angst vor dem Islam. Sie bedient sich der Vorstellung, dass der auf harter Arbeit beruhende freiheitlich-demokratische Staat durch eine äußere und fremde Kraft, den Islam, gefährdet werde. Dieser orientalistische Rassismus beschränkt sich nicht bloß auf die Behauptung der Überlegenheit der eigenen Gruppe, sondern fordert die Un-

7 | Liell, "Die Skandalisierung von Differenzen", S. 270.

8| Butterwegge, "Normalisierung der Differenz", S. 72.

9| Stecklina, wKleine Jungs mit zu großen Eiern'“, S. 82.

10 | Hauck, Die Gesellschaftstheorie und ihr Anderes, S. 48.

11 | Bukow, Feindbild: Minderheit, S. 66.

12 | Vgl. Chakrabarty, "Europa provinzialisieren“, S. 284ff.

13 | Said, Orientalismus, S. 14 
terdrückung und Beherrschung der anderen Gruppe durch Gesetze. Womit wir wiederum beim Beispiel des Kopftuches sind: Ein Effekt der aktuellen Konstruktion des »frauenfeindlichen muslimischen Mannes « und der »armen unterdrückten muslimischen Frau« ist die Einforderung von Sondergesetzen, welche die scheinbar defizitären Subjekte nach europäischen Maßstäben leiten und formen können.

\section{Wie der Westen die GeschlechtervorstelLUngen Kolonisiert}

Schon zu Zeiten des Kolonialismus galt es, die »indigene " Frau vor ihrer eigenen, frauenverachtenden Kultur zu retten. Die »zivilisatorischen" Eingriffe der Kolonialmacht wurden, wie Gayatri Chakravorti Spivak ausführt, mit der Vorstellung, »[w]eiße Männer retten braune Frauen vor braunen Männern ${ }^{14}$, legitimiert. Für die postkolonialen Debatten der Gegenwart rund um das Kopftuch und den Ganzkörperschleier gilt der Krieg in Afghanistan als zentrales Ereignis. Nebst der Bekämpfung der Terroristen wurde dieser als wichtiger Beitrag zur Befreiung der afghanischen Frauen angesehen. Dafür setzte sich nicht nur der damalige Präsident der Vereinigten Staaten, George W. Bush, sondern auch die First Lady, Laura Bush, ein. ${ }^{15}$ Im Gegensatz zu solchen direkten kolonialen Eingriffen, kann innerhalb von westlichen Gesellschaften von einem diskursiven Kolonialismus gesprochen werden, der sich aber, wie noch zu zeigen sein wird, auch in konkreten Praktiken, insbesondere im Bereich der Migrationspolitik, niederschlägt. ${ }^{16}$ Dabei handelt es sich um ein System, das auf ungleichen Machtverhältnissen, Hierarchien, Ethnozentrismen und andere Formen kultureller Dominanz beruht und diese reproduziert. Es kommt zur Auslöschung der Heterogenität der analysierten Subjekte, denen auch jegliche Art von aktiver Rolle oder Selbständigkeit abgesprochen wird: Sie werden zu analysierten Objekten. Die vermeintlichen Unterschiede werden der Kultur zugeschrieben, während historische und politische Fakten nicht nur nicht berücksichtigt, sondern als nicht relevant betrachtet werden. ${ }^{17}$ So wird zum Beispiel der Kontext der globalen Hegemonie der westlichen Wissenschaft, welche über Produktion, Publikation, Verbreitung und Konsum von Information und Ideen verfügt, nicht berücksichtigt und dadurch auch die direkte und aktive Machtausübung der Ersten Welt ignoriert. ${ }^{18}$

14 I Spivak, Can the subaltern speak?, S. 78. Auch damals schon spielten weiße Frauen, wie zum Beispiel Frauen von Kolonialherren oder Missionsfrauen, eine bedeutsame, wenn auch zweitrangige Rolle.

15 | Mahmood/Hirschkind, "Feminism, the Taliban, and Politics of Counter-Insurgency", S. 341.

16 Mohanty, Feminism Without Borders, S. 40.

17 | Abu-Lughod, "Do Muslim Women Really Need Saving?", S. 784

18 Mohanty, Feminism Without Borders, S. 21.
Ein signifikantes Beispiel für die westliche Deutungshegemonie ist die Konstruktion der nicht westlichen Frau. Wie Chandra Mohanty in »Feminism Without Borders« ausgeführt hat, werden »Dritte-Welt-Frauen« aus einer westlichen Perspektive als religiös (»nicht fortschrittlich»), familienorientiert (»traditionell«) unaufgeklärt (»sind sich ihrer Vernunft nicht bewusst《), unbelesen (»unwissend«) und häuslich (»rückständig «) definiert. ${ }^{19}$ Auch innerhalb feministischer und linker Kontexte wird die »Dritte-Welt-Frau« in einen Gegensatz zur westlichen emanzipierten Frau gesetzt. Durch die Konstruktion dieses spezifischen Unterschiedes, so Mohanty, vereinnahmen, reduzieren und kolonisieren westliche Feministinnen die Vielschichtigkeit der Lebenssituationen von nicht westlichen Frauen. ${ }^{20}$ Die Tatsache, dass unterschiedlichste Frauen als eine homogene Gruppe konstruiert, Geschlecht als einziger Ursprung von Unterdrückung angesehen und alle anderen strukturellen Machtverhältnisse ausgeblendet werden, zeigt die einseitige und für den postkolonialen Diskurs typische Betrachtungsweise auf. Konkret hat das zur Folge, dass die prekären Situationen von Migrantinnen ihrer spezifischen Kultur zugeschrieben werden. Für viele Frauen mit Migrationshintergrund sind es aber strukturelle und politische Hindernisse, welche ihnen den Zugang zu spezifischen Rechten verwehren. So wäre der Schutz vor häuslicher Gewalt für Migrantinnen in der Schweiz ein wichtigeres Thema als die momentan geführten Debatten zu Schleiern. Migrantinnen schrecken eher als ihre Schweizer Leidensgenossinnen davor zurück, sich von ihren gewalttätigen Ehemännern zu trennen, weil sie dadurch ihren Aufenthaltsstatus, welcher oftmals an die Männer gekoppelt ist, verlieren könnten.

In Frankreich war die Debatte über das Kopftuch sehr stark von der Instrumentalisierung der Geschlechtergerechtigkeit geprägt. Der einzige Weg zur Emanzipation der Frau wurde in einem Leben ohne Kopftuch gesehen. Gleichzeitig wurden soziale Probleme, vor allem in den Banlieues, von der Regierung und den Mainstreammedien auf die muslimische Kultur beschränkt, ohne dabei andere wichtige sozioökonomische und historische Faktoren, wie die koloniale Vergangenheit Frankreichs, zu beachten. Die Banlieues wurden zum Symbol und zum Beweis für die Kultur der »Anderen«. Elise Thiébaut, eine Feministin und ehemalige Kommunistin in Frankreich, scheute nicht davor zurück, das Kopftuch mit einer Vergewaltigung gleichzusetzen: »parler du voile, c'est parler du viol $\ll^{21}$. Diese undifferenzierte Haltung blendet aus, dass es Frauen gibt, die das Kopftuch aus eigenem Willen tragen. Verschiedene Studien haben gezeigt, dass das Kopftuch für viele junge Migrantinnen eine wichtige identitätsstiftende Rolle spielt und sich oftmals gegen die Bevormundung durch die Mehrheitsgesellschaft richtet. ${ }^{22}$ Solche Aspekte geraten aus dem Blick, wenn nicht muslimische europäische Menschen über muslimische

19 | Ebd., S, 40

20 | Ebd., S. 19.

21 | Thiébaut, "Tous voiles dehors", S. 1.

22 | Strahm, "Schleiersichten“, S. 12 
Frauen und ihre vermeintlich furchtbaren Lebenssituationen urteilen. Ein Großteil der Frauen, die sich in der öffentlichen Debatte zu Kopftuch und Ganzkörperverschleierung äußern, sind nicht muslimische Frauen, sondern Europäerinnen aus der Mittelklasse. Betroffene Muslimas hingegen kommen kaum zu Wort, und wenn doch, handelt es sich um immer dieselben »Vorzeigekopftuchträgerinnen«. Dadurch erfolgt die Diskussion über die muslimische Frau weitgehend aus einer Außenperspektive. Durch diese externe Beurteilung der Situationen muslimische Frauen können diese nicht als eigenständige und handlungsfähige Subjekte sichtbar werden, die ganz unterschiedliche Leben führen, differente Werte vertreten und in unterschiedlichsten Beziehungen zur (islamischen) Religion stehen. Gezeigt werden sie nur als Opfer, obwohl es gerade die diskursive Anordnung der Mehrheitsgesellschaft ist, die sie zu diesen Opfern stilisiert.

\section{ÜBer dIE SchWEIZER IDENTITÄT UND DIE ANDEREN}

Was in Frankreich schon seit längerem aufgrund der Kopftuchdebatten Thema war, begann in der Schweiz erst mit der Minarettinitiative, bei welcher die Gleichstellung der Geschlechter offensiv als Argument für eine rassistische Initiative benutzt wurde. Obwohl bereits vor der Minarettinitiative andere menschenverach tende Initiativen lanciert wurden, kam mit dieser spezifischen Initiative ein neues Phänomen auf. Die muslimische Frau stand plötzlich im Zentrum der öffentlichen Debatten. Was zuvor im Freundeskreis, im privaten Rahmen und vereinzelt diskutiert worden war, wurde nun zur Staatssache und zu einem Problem für die Allgemeinheit. Das Schema dieser Instrumentalisierung der muslimischen Frau sieht, ob in Frankreich oder in der Schweiz, ähnlich aus. Die männliche Dominanz und die Unterdrückung der Frau werden systematisch als muslimische Eigenschaften dargestellt. Die muslimische Kultur wird als sexistisch angesehen und die Unterdrückung der Frau zur muslimischen Angelegenheit erklärt. Dabei wird die freiheitlich-westliche Identität in den Debatten um die Minarettinitiative und nun aktuell um den Ganzkörperschleier konsequent den muslimischen Anderen gegenübergestellt. Deren Kultur wird als archaisch, sexistisch und äußerst gewalttätig konstruiert. Die muslimische Frau gilt als Opfer per se, der muslimische Ehemann, Vater oder Bruder als intrinsisch frauenfeindlich. Die ehemalige SVP-Nationalrätin Jasmin Hutter äußerte sich in ihrem Referat mit dem Titel »Rechtlose Frauen im Islam« folgendermaßen zur Rolle der muslimischen Frau:

"Allahs Töchter sind rechtlos. In den Westen eingewanderte Musliminnen stehen häufig unter dem Joch ihrer Männer. Allzu oft - faktisch eingesperrt in der eigenen Wohnung - auch hilflos der Gewalt ausgeliefert. Viele werden Opfer von Zwangsheiraten, allzu oft Ausgangspunkt für ein unfreies Leben in misslicher Lage.4${ }^{23}$

23 | Hutter, "Rechtlose Frauen im Islam".
Auch SVP-Nationalrat Dominique Baettig stellt »die Moslems« der »modernen westlichen Welt« gegenüber. ${ }^{24}$ Er spricht ausdrücklich über eine Kategorie Moslem, die »die positiven Errungenschaften unserer Kultur - Toleranz, Gleichstellung der Geschlechter, Respekt, Dialogbereitschaft $\aleph^{25}$ - destabilisieren würde. Die zur Beschreibung des Islams benutzten Adjektive sind klar negativ konnotiert. Die muslimische Kultur sei, im Gegensatz zu der gesicherten Geschlechtergleichheit in der Schweiz, männlich geprägt. Der Sexismus des Moslems wird als Bestandteil seiner Kultur und damit als gewöhnlich und alltäglich dargestellt. Diese Argumentation weist die Unterdrückung von Frauen den kulturell Anderen zu, während der Sexismus von nicht muslimischen Schweizer Männern dadurch außergewöhnlich und zur Ausnahme wird. Mit anderen Worten: Während der muslimische Mann qua seiner Kultur immer schon sexistisch ist, wird der Sexismus des Schweizers als ein individuelles Problem betrachtet, welches nichts mit seiner Kultur zu tun hat. Dieser Entlastungsdiskurs für westliche Männer führt dazu, dass das Problem der »Frauenunterdrückung« den Anderen zugeschoben wird und für sie kein Handlungsbedarf mehr zu bestehen scheint.

\section{Die Integration weisser Feministinnen in den Mainstream}

Für viele Feministinnen waren und sind solche islamophoben Debatten ein Ticket in den Mainstream. So konnten sich einige feministische Gruppen und Akteurinnen, die zuvor verpönt waren, durch die gegenwärtige Islamdebatte größeres Gehör und vermehrt Anerkennung verschaffen. Die zuvor in der Öffentlichkeit eher belächelten Feministinnen scheinen plötzlich ernst genommen zu werden - au Kosten allerdings von muslimischen Frauen. Beispielhaft für diese Entwicklung ist Alice Schwarzer, auf die im Folgenden kurz eingegangen werden soll. Schwarzer, deutsche Journalistin und Starfeministin par excellence, Verlegerin und Chefredakteurin der feministischen Frauenzeitschrift EMMA, gilt als eine führende Feministin im deutschsprachigen Raum und ist deshalb auch für die Schweiz von Bedeutung. Sie spricht sich neben einem »Burka«-Verbot auf der Straße auch für ein Kopftuchverbot an Schulen aus. Die muslimische Frau, welche einen Ganz. körperschleier trägt, beschreibt sie als ihrem Mann total unterwürfig. Den Begriff "freiwillig« setzt sie in Anführungs- und Schlusszeichen, um klar zu machen, dass es keine Frauen geben kann, die freiwillig einen Schleier tragen würden. Weiter sieht Schwarzer im Kopftuch die Bewegungsfreiheit und die Sicht der Frau eingeschränkt. Sie würde auf der Straße durch das Kopftuch gefährdet. Vor allem aber würde ihr Blick auf die Welt eingeschränkt. Sie tritt für ein Kopftuchverbot an Schulen ein, damit die Mädchen nicht als die »Anderen«stigmatisiert würden und

24 | Baettig, "Die Bedeutung der Initiative gegen den Bau von Minaretten«, Referat vom 08.07.2008.

25 | Ebd. 
eine Idee von wahrer Wahlfreiheit entwickeln könnten. Das Problem des Rassismus, der »die Anderen « erst als solche konstruiert, wird unsichtbar gemacht beziehungsweise an die betroffene Minderheit delegiert. In ihrem Pamphlet »Integration ist möglich«, das ein Auszug aus dem Abschlussbericht der Zukunftskommission $N R W$ ist, fordert Schwarzer eine Integration, die »auf der Basis unseres Rechtsstaates [...], inklusive der Gleichberechtigung der Geschlechter $\ll$ beruhe. ${ }^{26}$ Weiter geht sie darauf ein, dass in muslimischen Familien »die Mütter eingesperrt, die Töchter entrechtet und die Söhne verhetzt « würden. ${ }^{27}$ Die männlichen Mitglieder der muslimischen Gemeinschaft seien gefangen im Männlichkeitswahn, der mit Dominanz und Gewalt verknüpft wird. Deshalb fordert Schwarzer eine selbstbeDominanz und Gewalt verknüpft wird. Deshalb fordert Schwarzer eine selbstbeein Gewinn wären ${ }^{28}$ Ihre Darstellung muslimischer Familien geht ausschließlich von Extremen aus, die von Gewaltvorstellungen durchtränkt sind. Indem sie auf die Gewalt der muslimischen Kultur hinweist, agiert sie selbst gewalttätig, da sie die Menschen in starre Rollen drängt, fixiert und festschreibt. Ihre Argumentationsweise, welche das muslimische Gegenüber als unaufgeklärt darstellt und ihm jegliche Mündigkeit und Urteilsfähigkeit abspricht, ist typisch für einen kolonialen Feminismus, der sich über Frauen anderer Kulturen und Länder stellt. Die Gleichberechtigung, die Schwarzer anspricht, scheint für Frauen untereinander nicht zu gelten. Denn die freie Wahl, über ihren eigenen Körper zu entscheiden, bleibt der muslimischen Frau in dieser Argumentation verwehrt.

Wie aber kann diese Bevormundung von Musliminnen durch westliche $\mathrm{Fe}$ ministinnen erklärt werden? Ein kontroverser Befund könnte lauten, dass sich Europäerinnen zurzeit auch auf Kosten von Migrantinnen emanzipieren. In der Gegenüberstellung zur unterdrückten muslimischen Frau kann sich die westliche Frau in ihrer Selbstwahrnehmung als befreite und emanzipierte Frau bestärken. ${ }^{29}$ Paradox an diesem postkolonialen Blick auf die Andere ist, dass europäische Mittelschichtfrauen seit langem von Migrantinnen profitieren. Das beste Beispie dafür ist die Entlastung: Oft stellen doppelbelastete, berufstätige Frauen Migrantinnen für die Familien- und Hausarbeit ein. Die Arbeitsteilung und Hierarchie zwischen den Geschlechtern bleibt dabei unverändert, da sie durch eine Hierarchie zwischen einheimischen und zugewanderten Frauen kompensiert wird. Die chie zwischen einheimischen und zusgelagert und keineswegs gelöst. ${ }^{\circ \circ}$ Die europäische Frau kann sich in solchen postkolonialen Diskursen zudem zur Retterin der

26 | Schwarzer, Alice, "Integration ist möglich!", auf ihrer Homepage im April 2009. Siehe auch den Abschlussbericht der Zukunftskommission NRW: „Innovation und Soldarität“, April 2009: http://files1.derwesten.de/flashmm/PDF/zukunftskommission/Abschlussbericht.pdf? short=abschlussbericht

27 | Ebd.

28 | Ebd.

29 | Strahm, "Schleiersichten", S. 7.

30 | Lenzin, "Der andere Blick", S. 20.
Muslimin aufschwingen. Dorothee Wilhelm präzisiert in der Wochenzeitung richtig: "Besser zu wissen, was für Muslimas gut ist, als die diversen Muslimas selbst, ist sicher nicht feministisch, sondern Kolonialismus pur «. ${ }^{31}$ Eine problematische Folge dieser asymmetrischen Diskussionslage ist zudem, dass muslimische Frauen in eine Rechtfertigungssituation gestellt werden, statt dass sie eine Diskussion auf Augenhöhe führen können.

\section{STIMMEN GEGEN DIE ISLAMOPHOBIE}

Es gibt auch feministische Stimmen gegen den paternalistischen Diskurs über muslimische Frauen. Anhand von zwei Beispielen, der Kopräsidentin der SP Frauen Schweiz Julia Gerber Rüegg sowie des Interreligiösen Think-Tanks, sollen im Folgenden die Möglichkeiten und Grenzen dieser Positionen aufgezeigt werden.

Julia Gerber Rüegg richtet sich gegen die Instrumentalisierung von Geschlecht und kritisiert deshalb auch die Strategie der SVP. Diese, die sich als »Hüter der Frauenrechte« darstellt, appelliert nun plötzlich an Toleranz und Gleichstellung, hatte sich aber noch 2001 dagegen ausgesprochen, Vergewaltigungen in Ehen als Offizialdelikt zu betrachten - nicht zu reden von der aktuell geführten Antiabtreibungsdebatte. ${ }^{32}$ Deshalb sprach sich Rüegg vehement gegen das Minarettverbot aus und sieht auch keinen Grund, den Ganzkörperschleier zu verbieten, da es für ein solches Verbot in der Schweiz keinen Handlungsbedarf gebe. Sie betrachtet aber die »Burka« nichtsdestotrotz als eine Menschenrechtsverletzung. Die den Frauen in »extrem patriarchalen Gesellschaften aufgezwungene Burka« sei, so Julia Gerber Rüegg, »überall auf der Welt inakzeptabel«33. Der Zwang zur Verhüllung raube den muslimischen Frauen ihre Identität, schränke sie massiv in der Kommunikation ein und behindere schließlich »ihre Teilhabe an der Gesellschaft, Kultur und Politik «34. Stattdessen sollen sie sich auf »das Recht auf Selbstbestimmung, Sicherheit und Bildung «35 berufen können. Im Verlauf ihrer Argumentationen kommt es aber schließlich zu subtilem Rassismus, vor allem wenn sie sich mit dem Thema der Freiwilligkeit auseinandersetzt. So geht Rüegg zu Beginn zu Recht auf die materielle Abhängigkeit mancher Frauen ein, aufgrund derer sie sich nicht von ihren Männern trennen und emanzipieren würden. Aber auch sie erachtet die sogenannten kulturellen Unterschiede als Hauptproblem, anstatt sozioökonomische und politische Hindernisse zur Emanzipierung von Migrantinnen zu benennen. Problematisch ist der folgende Abschnitt aus ihrem Text »Freiwillig? «:

31 | Wilhelm, "Zum Schutz von Weib und Kind?", in: Wochenzeitung Online vom 03.12.2009.

32 | Gerber Rüegg, "Freiwillig?"

33 | Gerber Rüegg, "2010 - das Jahr der Frauenrechte".

34 | Ebd.

35 | Gerber Rüegg, "Freiwillig?". 
"Wenn ich vor der Güterabwägung stünde, unter der Knute, aber materiell gesichert und in meinem engeren Umfeld geachtet oder als allein stehende Frau frei, aber verstossen und im Elend zu leben, dann würde ich mich vermutlich auch ,freiwillig، verschleiern, in der Ehe ausharren und auf Befehl meine Beine spreizen. . $^{36}$

Der Schleier wird hier mit einer Ehe gleichgesetzt, in welcher die Frau ihrem Mann gänzlich ausgeliefert ist, und es wird nicht als möglich erachtet, dass der Schleier freiwillig getragen wird. Emanzipation, so Gerber Rüegg, sei mit Selbstbewusstsein und Chancen auf dem Arbeitsmarkt zu erreichen. Indem vorgegeben wird, strukturelle Probleme seien über das Selbstbewusstsein der betroffenen Migrantinnen zu lösen, wird die Problematik erneut auf die Eigenverantwortung der Migrantinnen abgeschoben. Dass auch Schweizer Bürgerinnen und Bürger zum Handeln aufgefordert sind, bleibt ausgeblendet. Zudem wird hier wiederum eine Verallgemeinerungstendenz sichtbar, da nicht jede Frau, die sich vornehmlich um den Haushalt kümmert, unglücklich mit ihrer Situation sein oder sich in einer Abhängigkeit befinden muss. Da die einzige »Emanzipationsmöglichkeit« von Migrantinnen oftmals darin besteht, als Putzfrau oder Hilfskraft zu arbeiten, gibt es genügend Frauen, welche die Arbeit als Hausfrau bevorzugen.

Zusammenfassend kann gesagt werden, dass die Hauptparolen von Julia Gerber Rüegg bezüglich der Frauen- und Menschenrechte zunächst überzeugend klingen, sich vor allem für Migrantinnen aber allzu oft als leeres Gerede entpuppen Das Argument der Selbstbestimmung stellt sich als Widerspruch heraus, da den Migrantinnen die Autonomie - etwa bei der Frage um den Ganzkörperschleier gerade abgesprochen wird. Verwendet wird also ein problematischer Begriff der Selbstbestimmung, der bereits vordefiniert ist. Unreflektiert bleibt auch, dass die Teilhabe an Politik, Gesellschaft und Kultur nicht nur aufgrund des Geschlechts, sondern auch wegen der sozialen und ethnischen Zugehörigkeit und fehlenden oder unzureichenden Papieren verunmöglicht wird. Auch wenn Julia Gerber Rüegg gute Ansätze in ihrer Politik verfolgt, wie beispielsweise die kantonale Standesinitiative zur Schaffung gesetzlicher Grundlagen, damit Jugendliche und junge Erwachsene ohne geregelten Aufenthalt eine Lehrstelle antreten können, vermag sie erstaunlicherweise nicht zu erkennen, welche rassistischen Mechanismen in ihrem eigenen Diskurs am Werk sind. Die weißen Frauen sind in dieser Emanzipationsgeschichte die Heldinnen und die muslimischen Frauen die Opfer.

Eine andere wichtige Stimme, welche sich gegen einen Feminismus ausspricht, »der die Stärken der eigenen Position auf der dunkel gemalten Folie der anderen entwickelt « $^{37}$, und sich fragt, wessen Interessen dieser Feminismus wirklich diene, ist der Interreligiöse Think-Tank, ein Zusammenschluss von Interessensvertreterinnen des interreligiösen Dialogs in der Schweiz, die gemeinsam gesellschaftliche und religionspolitische Fragen diskutieren. Sie mischen sich zudem in

\section{6 | Ebd.}

37 | Interreligiöser Think-Thank, "Offener Brief" die aktuellen religionspolitischen Debatten ein und entwerfen dazu neue Ansätze und wegweisende Ideen. ${ }^{38}$ In ihrem offenen Brief an Julia Onken, Schweizer Feministin und Psychologin, welche mit einem Rundbrief für die Annahme der Minarettinitiative geworben hatte, spricht sich der Interreligiöse Think-Tank im November 2009 ganz klar gegen eine im Namen der Frauenbewegung herrschende Bevormundung muslimischer Menschen durch Christen und Christinnen aus. In ihrer Schrift 16 Gründe für ein Nein, welche sich gegen die Minarettinitiative richtet, legen die Autorinnen dar, dass die Frauenfrage für politische Zwecke instrumentalisiert wird, dass die Definitionsmacht bezüglich des Islams nicht bei den Christinnen und Christen liegt und dass die Debatte Musliminnen und Muslime diskriminiere. 39 Neben dieser Analyse der Minarettinitiative ist der Think-Tank auch der Frage nachgegangen, wer in den öffentlichen Debatten zu Wort kommt. Dabei zeigte sich, dass, auch wenn die jeweiligen Verbote größtenteils von rechtskonservativer Seite ausgerufen und gefordert wurden, auch liberale und linke Vertreterinnen und Vertreter Verbote gefordert und die Debatten teilweise mit denselben Argumenten wie ihre politischen Gegner geführt haben. Auffallend ist, wer sich in der breiten Öffentlichkeit Gehör verschaffen konnte. Die Tendenzen der Mainstreammedien diesbezüglich sind ziemlich klar: Die größte Plattform wurde Frauen geboten, welche einen paternalistischen Diskurs verfolgten. Stimmen, welche sich für einen wirklichen Austausch und gegen eine Bevormundung aussprachen, wurden der breiten Öffentlichkeit vorenthalten. Vor allem Julia Onkens Rundbrief wurde in den Medien, in Schweizer Zeitungen und Fernsehsendungen, aufgenommen und somit auch weiterverbreitet. Kritische Stimmen zum Rundbrief und zur Initiative selbst, wie diejenige des Think-Tanks, wurden systematisch ignoriert und deshalb auch in der breiten Öffentlichkeit nicht gehört. Dabei wäre es wichtig gewesen, auch diese Stimmen zu hören, um zu zeigen, dass nicht alle Feministinnen in der Schweiz diesen paternalistischen und rassistischen Diskurs führen. Statt solche differenzierte und kritische Gegenstimmen aufzunehmen, hat etwa die Sendung Arena des Schweizer Fernsehens Alice Schwarzer eingeladen, die sich an dem populistischen Diskurs der Schweizer Mainstreammedien beteiligt. ${ }^{\circ}$

Allerdings bezieht sich auch der Interreligiosse Think-Tank auf problematische liberale Positionen. So beruft er sich zum Beispiel auf den schweizerischen Geist von Freiheit, Toleranz und humanitären Werten und bedauert, dass die Minarettinitiative diesen Werten widerspricht. An dieser Stelle wird auch die "gelebte Vielfalt verschiedener Kulturen, Sprachen und Religionen $\alpha^{41}$ der Schweiz zelebriert. Dabei wird allerdings vergessen, dass die Schweiz über regressive und repressive Aus-

38 | http://www.interrelthinktank.ch.

39 | Interreligiöser Think-Thank, "Anti-Minarett-Initiative".

40 | Arena, "Die Schweiz: mutig oder fremdenfeindlich?", ausgestrahlt am 11.12.2009, in: SF1-Videoportal http://www.videoportal.sf.tv/video?id=82ea94fe-4c1c-41c2-ad37-42b0 $27 \mathrm{~d} 80 \mathrm{~d} 5 \mathrm{e}$.

41 | Interreligiöser Think-Tank, "16 Gründe für ein Nein “. 
länder- und Asylgesetze verfügt und dass Schweizer Vorstellungen der Integration von Ausländern und Ausländerinnen vor allem Anpassung und Gehorsam beinhalten. Die Instrumentalisierung einer postkolonialen Geschlechterdebatte schläg sich auch bei Einbürgerungen nieder: So wurde im Jahre 2008 zwei Musliminnen die Schweizer Staatsangehörigkeit verwehrt, weil sie ein Kopftuch trugen. ${ }^{42}$

\section{Mitbestimmung Statt Integration}

Abschließend kann festgehalten werden, dass die vermeintlich antisexistischen Argumente in den gegenwärtigen Diskursen zur Situation muslimischer Frauen klar rassistisch sind, weil sexistische Praktiken als genuiner Bestandteil der muslimischen Kultur dargestellt werden. Musliminnen werden für diese Argumentation instrumentalisiert und dabei als unterwürfige und handlungsunfähige Frauen dargestellt. Ihnen gegenüber steht das Stereotyp des muslimischen Mannes, welcher seine Frau unterdrückt, zu Gewalt und Aggression neigt, deshalb nicht in die christlich-abendländische Tradition passt und somit nicht integrierbar sei. Nationale Grenzen, die durch Migration überwunden worden sind, werden in diesen postkolonialen Diskursen durch symbolische ersetzt. Gleichzeitig werden für Personen, die nicht aus der Europäischen Union stammen, die Aufenthaltsbestimmungen so verschärft, dass legale Migration kaum mehr möglich ist.

Ziel dieses Artikels ist es nicht zu behaupten, dass alle Frauen selbstbestimmt und freiwillig handeln würden, wenn sie zum Schleier greifen. Genauso wenig kann behauptet werden, dass westliche Frauen sich freiwillig der gängigen, tendenziell enthüllenden Mode anpassen. Freiwilligkeit und freie Wahl sind problematische und komplexe Themen, die ich an dieser Stelle nicht ausführlich behandeln kann. In diesem Beitrag ging es darum aufzuzeigen, dass die vermeintlich frauenbefreienden Debatten rund um das Thema Schleier oftmals rassistischer Natur sind. Sie greifen auf koloniale Bilder des Islams zurück und dienen dazu, eine Minderheit nicht bloß zu diffamieren, sondern sie auch zu beherrschen. Die Zurechtweisung von muslimischen Frauen durch neue Gesetze, welche ihr beispielsweise die Kleidung vorschreiben, findet in der Mehrheitsgesellschaft momentan viel Unterstützung. Das ist auch deshalb erstaunlich, weil ein Gesetz zur Bevormundung von Frauen ungute Erinnerungen wecken sollte: Der Schweizer Bundesstaat von 1848 war ein Männerstaat, der nur den Männern politische Rechte zuerkannte und den Frauen dienende und unterwürfige Rollen zuwies. Das alleinige Wahlrecht für Männer behielt bis 1971 , das alte Eherecht, welches die Handlungsfähigkeit der Frauen weiterhin einschränkte, bis zu den Gesetzesänderungen im Jahr 1988 ihre Gültigkeit. Auch die Einführung von Hosen war für Frauen in der Schweiz bis in die 1970er Jahre ein großes Thema; das Tragen von Hosen galt

42 | Kurt, "Die muslimische Frau mit Kopftuch", S. 58. vielerorts als Tabubruch. Diese wenigen Beispiele zeigen, wie unhaltbar die Dichotomie von der fortschrittlichen Schweiz und dem traditionellen Islam ist.

Das Analysieren, Verurteilen und Zurechtweisen von »fremden« Kulturen kann als Fortsetzung kolonialer Verhältnisse betrachtet werden, die sich nicht mehr vor Ort in den ehemaligen Kolonien, sondern im eigenen Land im Umgang mit Migranten und Migrantinnen ereignet. Wie sich bei der Minarettinitiative gezeigt hat und wie sich mit der »Burka«-Debatte abzeichnet, bedeutet Integration in der Schweiz vor allem, alles gleichzuschalten. Von Mitbestimmung der Migrantinnen und Migranten ist kaum die Rede. Das ist allerdings nur eine Strategie: Zwar wird behauptet, dass die Bevölkerung homogenisiert und identisch gemacht werden soll, die wirkliche Angleichung bleibt den Migrantinnen und Migranten aber verwehrt. Die Anderen werden immer auf ihr Anderssein zurückgeworfen. Obwohl Assimilation gefordert wird, dienen diese Debatten dazu, Differenz herzustellen. Die fremdenfeindlichen Debatten, die mit dieser Differenz operieren, haben zum Ziel, von den wirklichen Problemen abzulenken. Wenn die Ausländerpolitik der Schweiz in den letzten Jahrzehnten betrachtet wird, vor allem die Debatten um die Überfremdung, das Asylrecht und um Ausländerinnen und Ausländer im Allgemeinen, dann ist der gegenwärtige Diskurs über die angeblich muslimische Eigenart nicht überraschend. Fremde sind unerwünscht, als Sündenbock aber willkommen. Sie werden als Symbol der Bedrohung und Verunsicherung politisch instrumentalisiert. Die Kategorien ändern sich zwar - von den Italienern über die Türkinnen und die Jugoslawinnen zu den Muslimen -, doch die Absicht und der Inhalt von fremdenfeindlichen Botschaften bleiben gleich: die Abgrenzung des »Eigenen « gegenüber dem »Anderen ${ }^{43}$ Anstatt die Hürden beim Zugang zu Bildung, Arbeit und politischen Rechten abzubauen, wird die sogenannte Integrationsunwilligkeit den Migrierten zugeschrieben. ${ }^{44}$ Die vermeintlich nicht $\mathrm{zu}$ vereinbarenden kulturellen Unterschiede dienen dazu, die immer restriktiveren Ausländergesetze, die Schließung der Schweizer Grenzen und die Etablierung der Festung Europa zu begründen. Dabei spielt Geschlecht eine bedeutsame Rolle: Durch den vorgeschobenen Grund der patriarchalen Unterdrückung gelingt es, die zunehmende Illegalisierung von Migrantinnen und Migranten zu erwirken. Für die Situation muslimischer Frauen, um deren Wohl sich angeblich so viele Schweizerinnen und Schweizer sorgen, birgt diese Entwicklung rein gar keine Vorteile.

43 | Buomberger, Kampf gegen unerwünschte Fremde, S. 29.

44 | Bühlmann, "Die Burkadebatte als Lehrstücka, S. 26. 


\section{LITERATURVERZEICHNIS}

Abu-Lughod, Lila, "Do Muslim Women Really Need Saving? Anthropological Reflections on Cultural Relativism and Its Others«, in: American Anthropologist, Jg. 104, H. 3 (2002), S. $783-790$.

Baspinar, Deniz, »Burkaverbot, einig Vaterland«, in: Die Zeit Online vom 01.05.2010, http://www.zeit.de/gesellschaft/zeitgeschehen/2010-05/belgien-burkaverbotkommentar.

Baettig, Dominique, »Die Bedeutung der Initiative gegen den Bau von Minaretten«, Referat vom 08.07.2008, http://www.minarette.ch/referate/archiv-2008/ index.html, 16.01.2012.

Bühlmann, Cécile, »Die Burkadebatte als Lehrstück für die Instrumentalisierung von Frauenrechten«, in: Olympe. Feministische Arbeitshefte zur Politik, Jg. $17 \mathrm{H}$. 31 (2010), S. 21-28.

Bukow, Wolf-Dietrich, Feindbild: Minderheit. Ethnisierung und ihre Ziele, Opladen 1996.

Bukow, Wolf-Dietrich/Nikodem, Claudia/Schulze, Erika/Yildiz, Erol (Hg.), Was heißt hier Parallelgesellschaft? Zum Umgang mit Differenzen, Wiesbaden 2007.

Buomberger, Thomas, Kampf gegen unenü̈nschte Fremde - Von James Schwarzenbach bis Christoph Blocher, Zürich 2004.

Butterwegge, Christoph, »Normalisierung der Differenz oder Ethnisierung der sozialen Beziehungen?«, in: Bukow, Wolf-Dietrich/Nikodem, Claudia/Schulze, Erika/Yildiz, Erol (Hg.), Was heißt hier Parallelgesellschaft? Zum Umgang mit Differenzen, Wiesbaden 2007, S. 65-80.

Chakrabarty, Dipesh, »Europa provinzialisieren. Postkolonialität und die Kritik der Geschichte«, in: Conrad, Sebastian/Randeria, Shalini (Hg.), Jenseits des Eurozentrismus. Postkoloniale Perspektiven in den Geschichts- und Kulturwissenschaften, Frankfurt a.M/New York 2002, S. 283-312.

Gerber Rüegg, Julia, »2010 - das Jahr der Frauenrechte«, vom o6.12.2010, http:// www.juliagerber.ch/files/JahrFrauenrechte.pdf, 27.07.2011.

Gerber Rüegg, Julia, »Freiwillig?«, http://www.juliagerber.ch/files/Freiwillig.pdf, vom 28.04.2010.

Hauck, Gerhard: Die Gesellschaftstheorie und ihr Anderes. Wider den Eurozentrismus der Sozialwissenschaften, Münster 2003.

Hollenstein, Pascal, »Widmer-Schlumpf erwägt Burka Verbot«, in: Neue Zürcher Zeitung Online vom 08.11.2009, http://www.nzz.ch/nachrichten/schweiz/wid mer-schlumpf_erwaegt_burka-_verbot_fuer_musliminnen_1.3985315.html.

Hutter, Jasmin, »Rechtlose Frauen im Islam«, http://www.minarette.ch/ref_og. html, 09.05.2007.

Dies., »Scharia und Minarett aus Sicht der Frau«, http://www.minarette.ch/ref_o3. html, 08.07.2008.
Interreligiöser Think-Tank, »16 Gründe für ein Nein«, http://www.interrelthink tank.ch/news_list.php? mastertable=category_news_page\&masterkeyı=2, 09.11 .2009 .

Interreligiöser Think-Tank »Anti-Minarett-Initiative«, http://www.interrelthink tank.ch/news_list.php?mastertable=category_news_page\&masterkeyı=3, 20.05.2010.

Ders. »Offener Brief an Julia Onken«, http://www.interrelthinktank.ch/news_list. php? mastertable=category_news_page\&masterkeyl=2, 18.11 .2009 .

Kurt, Stefanie Tamara, »Die muslimische Frau mit Kopftuch im Spannungsverhältnis zum Diskriminierungsverbot«, in: Olympe. Feministische Arbeitshefte zur Politik, Jg. 17, H. 31 (2010), S. 58-65.

Lenzin, Rifa'at, »Der andere Blick: Die Genderfrage aus islamischer Sicht«, in: Olympe. Feministische Arbeitshefte zur Politik, Jg. 17, H. 31 ( 2010), S. 14-20.

Liell, Christoph, »Die Skandalisierung von Differenzen. Das Beispiel ethnisierter Jugendgewalt«, in: Bukow, Wolf-Dietrich/Nikodem, Claudia/Schulze, Erika/ Yildiz, Erol (Hg.), Was heißt hier Parallelgesellschaft? Zum Umgang mit Differenzen, Wiesbaden 2007, S. 269-285.

Mahmood, Saba/Hirschkind, Charles, »Feminism, the Taliban, and Politics of Counter-Insurgency«, in: Anthropological Quarterly, Jg. 75, H. 2 (2002), S. 339354 .

Mohanty, Chandra Talpade, Feminism Without Borders - Decolonizing Theory, Practicing Solidarity, Durham 2003.

N. N., "Burkaverbot wird Abstimmungsthema«, in: Neue Zürcher Zeitung Online vom 19.05.2011, http://www.nzz.ch/nachrichten/politik/schweiz/burkaverbot_ abstimmung_tessin_initiative_ghiringhelli_1.10637440.html, 25.01.2012.

Said, Edward W., Orientalismus, Frankfurt a.M. 1981.

Schwarzer, Alice, »Integration ist möglich!«, auf ihrer Homepage April 2009, http://www.aliceschwarzer.de/publikationen/aliceschwarzer-artikel-essays/ integration-ist-mglich-2009/, 16.01.2012.

Schwarzer, Alice, »Für ein Burka-Verbot!«, in: Frankfurter Allgemeine Zeitung Online vom 20.07.2010, http://www.aliceschwarzer.de/publikationen/aliceschwarzer -artikel-essays/fuer-ein-burka-verbot.

Spindler, Susanne, Corpus delicti. Männlichkeit, Rassismus und Kriminalisierung im Alltag jugendlicher Migranten, Münster 2006.

Spivak, Gayatri Chakravorty, Can the subaltern speak? Postkolonialität und subalterne Artikulation, Wien 2008

Stecklina, Gerd, »Kleine Jungs mit zu großen Eiern<. Männlichkeitsstereotype über junge männliche Migranten«, in: Gemende, Marion/Munsch, Chantal/ Weber-Unger Rotino, Steffi (Hg.), Eva ist emanzipiert, Mehmet ist ein Macho, München 2007, S. 74-90.

Strahm, Doris, »Schleiersichten - Feministische Debatten um das Kopftuch, Geschlechterkonzepte und Religion« (2007), http://www.doris-strahm.ch/ Strahm_4_02.pdf, 27.07.2011. 
Thiébaut, Elise, »Tous voiles dehors«, http://sisyphe.org/spip.php?article761, 11.11 .2003 .

Wilhelm, Dorothee, »Zum Schutz von Weib und Kind?«, in: Wochenzeitung Online vom 03.12.2009, http://www.woz.ch/artikel/2009/nr49/schweiz/18676.html, 25.01 .2012 .

\section{Ruanda, Trinidad und Co.}

Koloniale Verstrickungen und postkoloniale Aufbrüche

in der Schweizer Gegenwartsliteratur

Alexander Honold

\section{DiE SCHWEIZ UND IHR POSTKOLONIALES INTERESSE. VERÄNDERUNGEN DER LITERARISCHEN AGENDA}

»Als Schweizer«, so glaubt der Mitarbeiter einer Entwicklungshilfeorganisation in Kigali, Ruanda, »habe ich mit dem Kolonialismus nichts zu tun «. ${ }^{1}$ Mit diesem Ausspruch und der dahinterstehenden Haltung, die Lukas Bärfuss dem Protagoniston seines Romans Hundert Tage zuschreibt, trifft der Autor den Nagel auf den Kopf. Kaum eine Nation ist der kolonialisierenden Machtausübung so unverdächtig, kaum eine genießt ein traditionell so stark auf humanitäre Zielsetzungen ausgerichtetes Image wie diejenige, deren Vertreter unter der Flagge des weißen Kreuzes auf rotem Grund agieren. Warum dann überhaupt die heikle Präsenz vor Ort, in einem Krisengebiet? Um großzügig und aus überlegener Position den Benachteiligten, Notleidenden, Kriegs- und Gewaltopfern zu helfen, natürlich. Aber auch, um im gleichen Zug auf »unzuständig« plädieren zu können, wenn die aus der kolonialen Vergangenheit herrührenden, nur mühsam zurückgedrängten Konfliktherde zerstörerisch wieder aufflammen.

Schaut man sich aber das Umfeld an, in dem der junge Mann agiert, um den es in Bärfuss' Roman geht, vergegenwärtigt man sich zudem die geschilderte Arbeitsweise der Schweizer Kollegen und ihr hierarchisches Verhältnis zu den afrikanischen Mitarbeitern, Helfern und Domestiken, betrachtet man schließlich die in sich widersprüchliche und aggressive sexuelle Beziehung zwischen dem Schweizer Mann und seiner ruandischen Geliebten, dann wiegt dieses »nichts «, mit dem der Protagonist die Frage nach dem Kolonialismus abtut, auf einmal viel schwerer. Der Kontext nämlich, in dem die eingangs zitierte, abwimmelnde Bemerkung fällt,

1 Bärfuss, Hundert Tage, S. 126. 